DOI: https://doi.org/10.14311/TPFM.2021.014

\title{
NUMERICAL TESTS OF VANISHING DIFFUSION STABILIZATION IN OLDROYD-B FLUID FLOW SIMULATIONS
}

\author{
Marília Pires ${ }^{1,2}$, Tomáš Bodnár 3,4 \\ ${ }^{1}$ Department of Mathematics, Technology Sciences School, University of Évora, \\ Rua Romão Ramalho, 7000-671, Évora, Portugal \\ 2 CIMA-UE, CEMAT-IST \\ 3 Institute of Mathematics, Czech Academy of Sciences, \\ Žitná 25, 11567 Prague 1, Czech Republic \\ ${ }^{4}$ Faculty of Mechanical Engineering, Czech Technical University in Prague, \\ Karlovo Náměstí 13, 12135 Prague 2, Czech Republic
}

\begin{abstract}
This work presents some numerical tests of finite element solution of incompressible Oldroyd-B fluids flows, using different types of numerical stabilization. In this study the diffusive term (Laplacian of extra stress) is added to the tensorial constitutive relation where it is multiplied by a coefficient, that is variable in time. The goal is to make this diffusion coefficient vanish in time, so that the final solution remains unaffected by the added diffusion term.

A series of numerical tests was performed for the steady two-dimensional Oldroyd-B fluid flow in corrugated channel (tube) to compare different versions of the vanishing stabilization terms and assess their efficiency in enforcing the solution convergence, without affecting the final steady state.
\end{abstract}

Keywords: Finite Element Method, Oldroyd-B Fluid, Numerical Stabilization, Stress Diffusion.

\section{Introduction}

The motivation for the development of this study comes from our previous work [13], where the numerical results clearly proved the advantage of the numerical method stabilized by the addition of the diffusive term proportional to the time derivative of the Laplacian of the elastic stress tensor $\left(\alpha \cdot \Delta\left(\tau_{h}^{n}-\tau_{h}^{n-1}\right)\right)$.

In this work we are looking for an alternative artificial diffusion term that will disappear during the iterative process, expecting that the final converged steady solution will be not affected by the added (but finally vanished) artificial diffusion. Several possibilities of the type $\alpha(t) \Delta \tau_{h}^{n}$ are proposed, where $\alpha(t)$ is a function, depending on time (vanishing in time).

The aim of this short contribution is to present some of the effects of the diffusive stabilization on the solution. The simulations with constant and vanishing stabilization are compared with results obtained without stabilization. At this stage the focus is rather on qualitative changes in the obtained solution, rather than in reaching higher critical Weissenberg numbers.

\section{Mathematical Model}

The unsteady, incompressible, isothermal viscoelastic flow of homogeneous Oldroyd-B fluid is considered in a bounded domain $\Omega \subset \mathbb{R}^{d}(d=2,3)$ over the time interval $\left[0, T_{f}\right]\left(T_{f}>0\right)$. The governing system of equations can be written in dimensionless form as:

$$
\left\{\begin{array}{l}
\mathcal{R} e\left(\frac{\partial \boldsymbol{u}}{\partial t}+\boldsymbol{u} \cdot \nabla \boldsymbol{u}\right)+\nabla p=2(1-\eta) \nabla \cdot \mathbf{D}+\nabla \cdot \boldsymbol{\tau}+\mathbf{f} \\
\nabla \cdot \boldsymbol{u}=0 \\
\boldsymbol{\tau}+\mathcal{W} e\left(\frac{\partial \boldsymbol{\tau}}{\partial t}+\boldsymbol{u} \cdot \nabla \boldsymbol{\tau}-\nabla \boldsymbol{u}^{T} \cdot \boldsymbol{\tau}-\boldsymbol{\tau} \cdot \nabla \boldsymbol{u}\right)=2 \eta \mathbf{D}
\end{array}\right.
$$

where $\mathcal{R} e=\frac{\boldsymbol{U} L}{\nu}$ and $\mathcal{W} e=\frac{\lambda \boldsymbol{U}}{L}$ are the Reynolds and Weissenberg numbers, respectively. The symmetric tensor $\boldsymbol{\tau}$ is the viscoelastic contribution to the extra stress tensor given by $\mathbf{T}=2 \nu_{\star} \mathbf{D}+\boldsymbol{\tau}$, 
being $\mathbf{D}=\frac{1}{2}\left(\nabla u+\nabla u^{T}\right)$ the strain tensor (symmetric part of velocity gradient). Depending on the stress model adopted, $\nu_{\star}$ can either represent the viscosity of the solvent (for the polymer solution) or part of the total (apparent) stress viscosity of the system. Here $\eta$ is such that $\nu=\nu_{\star}+\eta$, and $\nu$ is the total kinematic viscosity. The body force is denoted by $\mathbf{f}$.

\section{$3 \quad$ Numerical Method}

The problem (1) is complete, prescribing, over the boundary $\partial \Omega=\Gamma_{\text {in }} \cup \Gamma_{w} \cup \Gamma_{\text {out }}$ of the domain $\Omega$, the initial and boundary conditions by:

$$
\begin{cases}\boldsymbol{u}=0 & \text { on } \Gamma_{w} \\ \boldsymbol{u}=u_{i n} & \text { on } \Gamma_{i n} \\ \boldsymbol{\tau}=\boldsymbol{\tau}_{i n} & \text { on } \Gamma_{i n} \\ \boldsymbol{u}_{\mid t=0}=u_{0} & \end{cases}
$$

The problem (1-2) is numerically solved using the so-called reference viscosity scheme. The extra stress tensor is split according to $\mathbf{T}=2 \eta \mathbf{D}+\boldsymbol{\tau}$ into the viscoelastic stress $\boldsymbol{\tau}$ and the viscous part $\boldsymbol{\tau}_{s}=2 \eta \mathbf{D}$. The parameter $\eta \in[0,1]$ is, in this case, the dimensionless polymer viscosity contribution. This splitting allows to decouple the kinematic and the non-Newtonian viscoelastic stress even though the divergence of $\boldsymbol{\tau}$ is included in the momentum equations as a pseudo-body force while the constitutive equation includes a contribution from the Newtonian part.

The momentum equation is discretized following the Rothe Method. This means that the discretization in space is done after the discretization in time. The mixed finite element method is used to discretization with respect to space, as it satisfies the compatibility condition known as the discrete LBB (Ladyzheskaya, Babuška and Brezzi) or inf-sup condition [5], [9], [14] or [15].

The Characteristic Galerkin Method evaluates time derivatives of vector field on a Lagrangian frame using the characteristic lines or trajectories describing a material particle when it has been driven by the field at the velocity of the field [14]. More details of the discretization of the model (1), by characteristic finite element method, can be found in our previous work [13].

\subsection{Discretization details}

On the time interval $\left[0, T_{f}\right]$ a set of points (time instants) $t_{n}$ is defined by $t_{n}=n \Delta t=n \frac{T_{f}}{N}$, $n=0, \ldots, N$. The $v^{n}$ denotes the approximation of $v$ at the time instant $t_{n}$, i.e., $v^{n} \approx v\left(t_{n}, \cdot\right)$. Consider $x \in \Omega$.

To discretize the momentum and constitutive equations with respect to time (semi-discretization), the implicit Euler scheme was associated with Characteristic Galerkin Method. Hence, the convective time derivative is approximated as:

$$
\frac{D v}{D t}=\frac{\partial v}{\partial t}+\boldsymbol{u} \cdot \nabla v \approx \frac{v^{n}-v^{n-1} \circ X^{n-1}}{d t}
$$

where $X$ is the convecting field defined by $X(x)=x_{t}$ with the particle path $x_{t}$ being the solution of $\dot{x}_{t}=u\left(x_{t}\right), x_{t=0}=x$ and $v \circ X(x)=v(x-u(x) t)=v^{n-1}\left(x^{\star}\right) \equiv v_{\star}^{n-1}$, with $x^{\star}$ the position at time $t_{n-1}$ of the particle located at $x$ at time $t_{n}$.

The convergence, stability and other properties of this kind of discretization can be found in literature, e.g. in [9].

To discretize the governing equations with respect to space, the $P_{2}-P_{1}$ finite elements were considered on a non-degenerated mesh $\mathbb{T}_{h}$ of diameter $h$, which defines the numerical domain $\Omega_{h} \subseteq \Omega \subset \mathbb{R}^{2}$, whose boundary is $\partial \Omega_{h}=\Gamma_{h, \text { in }} \cup \Gamma_{h, w} \cup \Gamma_{h, \text { out }}$.

The resulting discretization of the variational problem defined by (1-2) reads: For each $t \in\left[0, T_{f}\right],\left(u_{0, h}, \boldsymbol{\tau}_{0, h}\right) \in X_{h} \times \mathcal{S}_{h}$, find $\left(\boldsymbol{u}_{h}, p_{h}, \boldsymbol{\tau}_{h}\right) \equiv\left(u_{h}(t, \cdot), p_{h}(t, \cdot), \boldsymbol{\tau}_{h}(t, \cdot)\right) \in X_{h} \times M_{h} \times \mathcal{S}_{h}$ such that 


$$
\begin{cases}\int_{\Omega} 2(1-\eta) \mathbf{D}_{h}^{n}: \nabla \mathbf{v}_{h}+\mathcal{R} e \int_{\Omega} \frac{\boldsymbol{u}_{h}^{n}-\boldsymbol{u}_{\star, h}^{n-1}}{\Delta t} \cdot \mathbf{v}_{h}-\int_{\Omega} p_{h}^{n} \nabla \cdot \mathbf{v}_{h}=\int_{\Omega}\left(\nabla \cdot \boldsymbol{\tau}_{h}\right) \mathbf{v}_{h}, & \forall \mathbf{v}_{h} \in X_{h} \\ \int_{\Omega}\left(\nabla \cdot \boldsymbol{u}_{h}^{n}\right) q_{h}=0, & \forall q_{h} \in M_{h} \\ \int_{\Omega}\left(\boldsymbol{\tau}_{h}^{n}+\mathcal{W} e \frac{\boldsymbol{\tau}_{h}^{n}-\boldsymbol{\tau}_{\star, h}^{n-1}}{\Delta t}\right): \mathbf{S}_{h}=\int_{\Omega}\left[2 \eta \mathbf{D}_{h}+\mathcal{W} e\left(\nabla \boldsymbol{u}_{h}^{T} \cdot \boldsymbol{\tau}_{h}^{n}+\boldsymbol{\tau}_{h}^{n} \cdot \nabla \boldsymbol{u}_{h}\right)\right]: \mathbf{S}_{h}, & \forall \mathbf{S}_{\mathbf{h}} \in \mathcal{S}_{h}\end{cases}
$$

where $X_{h}=\left\{\mathbf{v}_{h} \in C(\bar{\Omega}) \cap \mathbf{H}^{1}(\Omega): \mathbf{v}_{h}=0\right.$ on $\Gamma_{h}, w, \mathbf{v}_{h}=u_{i n}$ on $\Gamma_{h, i n}$ and $\left.\mathbf{v}_{h_{\mid}} \in \mathbb{P}_{2}(\mathcal{T}), \forall \mathcal{T} \in \mathbb{T}_{h}\right\}$,

$M_{h}=\left\{q_{h} \in C(\bar{\Omega}) \cap L_{0}^{2}(\Omega): q_{\left.\right|_{\mid}} \in \mathbb{P}_{1}(\mathcal{T}), \forall \mathcal{T} \in \mathbb{T}_{h}\right\}$ and

$\mathcal{S}_{h}=\left\{\mathbf{S}_{h} \in \mathbf{C}(\bar{\Omega}) \cap\left[L^{2}(\Omega)\right]^{2 \times 2}: \mathbf{S}_{h}^{T}=\mathbf{S}_{h}\right.$ and $\left.\left.\mathbf{S}_{h, i j}\right|_{\mathcal{T}} \in \mathbb{P}_{2}(\mathcal{T}), \forall \mathcal{T} \in \mathbb{T}_{h}\right\}$

\subsection{Solution algorithm}

The problem defined by (3) can be solved by a decoupled iterative algorithm (Picard method) even when the steady solution is searched. Based on the unsteady formulation and the time-marching procedure, the stationary state can be attained as the long-time limit solution of the evolutionary model complemented by the steady boundary conditions. If the solution converges in time, the stationary state is obtained.

The linear system associated to discrete Stokes problem is solved by Crout scheme and the linear system associated to the other equations is solved by a multi-frontal Gauss LU factorization implemented in the FreeFem++ [7], [8], package UMFPACK.

\subsection{Stabilization technique}

In our previous work [13], we proposed an extra diffusive term

$$
\alpha \cdot \Delta\left(\tau_{h}^{n}-\tau_{h}^{n-1}\right)
$$

to be added to the constitutive equation, to stabilize the simulations for high Weissenberg numbers.

This term (4) can be seen as a constant coefficient $\alpha$ times the Laplacian of time-derivative of the tensor $\boldsymbol{\tau}$, i.e. of the form $\alpha \cdot \Delta \boldsymbol{\tau}_{t}$. This term will vanish when $\boldsymbol{\tau}_{h}^{n} \rightarrow \boldsymbol{\tau}$, i.e. when the numerical solution will converge to the steady state. The constant coefficient $\alpha$ represents a diffusive parameter for the elastic stress tensor.

The numerical tests showed that the term (4) stabilizes the simulations, independently of the choice the constant $\alpha$. It means that as $\alpha \cdot \Delta\left(\tau_{h}^{n}-\tau_{h}^{n-1}\right) \rightarrow 0$ when the solution converged to steady state. Apparently, the solution obtained corresponds to the solution of the original problem without diffusion. This however is not the case of the standard diffusion term $\alpha \cdot \Delta \boldsymbol{\tau}$, where the diffusive term doesn't vanishes and the obtained solution always corresponds to another, diffusive, model.

Our long term goal is to stabilize the simulations for high Weissenberg numbers. But we keep in mind the theoretical questions related to proof of well-posedness of the problem. This is why in this work we propose an alternative (simpler) vanishing diffusive term for the constitutive equation. Instead of $\alpha \cdot \Delta \boldsymbol{\tau}_{t}$ with $\alpha$ constant in time, we propose multiple variants of $\alpha(t) \Delta \boldsymbol{\tau}$ with a function $\alpha(t)$ depending on time, vanishing when $t \rightarrow \infty$.

This case is equivalent to take the constitutive equation in the form

$$
\boldsymbol{\tau}+\mathcal{W} e\left(\frac{\partial \boldsymbol{\tau}}{\partial t}+\boldsymbol{u} \cdot \nabla \boldsymbol{\tau}-\nabla \boldsymbol{u}^{T} \cdot \boldsymbol{\tau}-\boldsymbol{\tau} \cdot \nabla \boldsymbol{u}\right)=2 \eta \mathbf{D}+\alpha(t) \Delta \boldsymbol{\tau}
$$

for which the weak formulation given by

$$
\int_{\Omega}\left[\boldsymbol{\tau}+\mathcal{W} e\left(\frac{\partial \boldsymbol{\tau}}{\partial t}+\boldsymbol{u} \cdot \nabla \boldsymbol{\tau}\right)\right]: \mathbf{S}=\int_{\Omega}\left[2 \eta \mathbf{D}+\mathcal{W} e\left(\nabla \boldsymbol{u}^{T} \cdot \boldsymbol{\tau}+\boldsymbol{\tau} \cdot \nabla \boldsymbol{u}\right)\right]: \mathbf{S}-\alpha \nabla \boldsymbol{\tau}: \nabla \mathcal{S}, \quad \forall \mathbf{S} \in \mathcal{S}
$$


where $\mathcal{S}=\left\{\mathbf{S} \in\left[L^{2}(\Omega)\right]^{2 \times 2}: \mathbf{S}^{T}=\mathbf{S}\right\}$.

The approximate finite element problem for the tensor can be reformulated as:

For each $t \in\left[0, T_{f}\right], \boldsymbol{\tau}_{0, h} \in S_{h}=\left[\mathcal{M}_{h}\right]^{2 \times 2}$, find $\boldsymbol{\tau}_{h} \in \mathcal{S}_{h}$ such that each component $\boldsymbol{\tau}_{h, i j}$ verifies

$$
\int_{\Omega}\left(\boldsymbol{\tau}_{h, i j}^{n}+\mathcal{W} e \frac{\boldsymbol{\tau}_{h, i j}^{n}-\boldsymbol{\tau}_{\star, h, i j}^{n-1}}{\Delta t}\right): \mathbf{S}_{h, i j}=\int_{\Omega}\left[2 \eta \mathbf{D}+\mathcal{W} e\left(\nabla \boldsymbol{u}^{T} \cdot \boldsymbol{\tau}_{h}^{n}+\boldsymbol{\tau}_{h}^{n} \cdot \nabla \boldsymbol{u}\right)\right]_{i j}: \mathbf{S}_{h, i j}-\alpha(t) \nabla \boldsymbol{\tau}_{h, i j}^{n}: \mathbf{S}_{h, i j}
$$

for all $\mathbf{S}_{h} \in \mathcal{S}_{h}$.

\section{Numerical Simulations}

The above described model and algorithm is implemented using the FreeFem $++[7]$ toolboxes. The numerical simulations presented hereafter are performed for a simple corrugated channel, for which the limits of the code become apparent.

All the tests are performed by considering the dimensionless elastic viscosity parameter $\eta=0.1$. For fixed $\mathcal{R} e=1000$, the continuation method is applied with respect to $\mathcal{W} e$, which means, the Weissenberg number is increased incrementally, while taking the converged solution obtained for lower $\mathcal{W} e$ as an initial condition for higher $\mathcal{W} e$ simulation.

\section{Computational domain}

The corrugated pipe is assumed to consist of (sufficienly long) straight inlet and outlet parts with dimension (width/height) $D$. The attached contraction/expansion parts have length $L_{c o n}, L_{\text {exp }}$. Between these parts, several identical segments are inserted. These segments are characterised by their minimum and maximum diameters $D_{\min }$ resp. $D_{\max }$ and the length $L_{\text {seg }}$. All the curvilinear walls have sinusoidal shape.

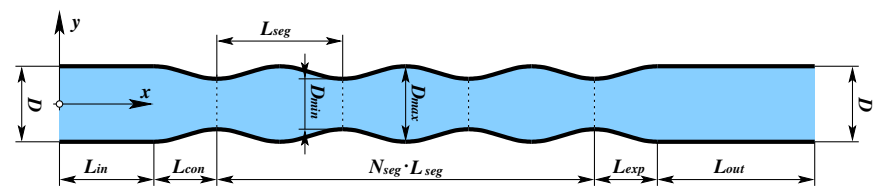

Figure 1: Geometry definition for the corrugated pipe case.

To generate the grids used in the presented simulations, each unit of length on the boundary is first divided into 10 equal segments and then the (almost regular) grid is obtained for the interior of the domain. The grid is generated by FreeFem++, using the Delaunay-Voronoi algorithm.

\section{Boundary conditions}

The inlet and outlet straight parts of the channel (see Fig.1) allow to consider the flow on the respective boundaries to be fully developed. On the upstream boundary the analytical Poisseuille solution for the fully developed flow in a straight channel for the respective model is imposed (analytical solution is available). On the solid wall no-slip conditions are used for the fluid velocity.

\section{Artificial diffusion setup}

In this work we chose two different families of functions $\alpha(t)$ such that $\alpha(0)=10^{-4}$ and then it monotonically decays $\alpha(t) \rightarrow 0$ when $t \rightarrow 0$. The starting value $\alpha(0)=10^{-4}$ was chosen to compare the results with the constant diffusion case and also with the previous work shown in [13]. The chosen functions $\alpha(t)$ decay at different rate as it is shown in the Fig. 2.

\subsection{Numerical results}

The above described test case of flow of a viscoelastic fluid through the 2D corrugated channel was solved for a range of Weissenberg numbers. The aim of the performed numerical simulations was twofold. First to find function $\alpha(t)$ that decays properly (not too fast to stabilize, not too slow 


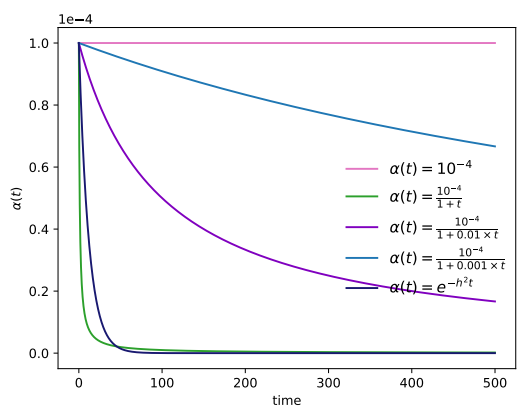

Figure 2: Behavior of the function $\alpha(t)$ (diffusion coefficient) with respect to time

to vanish) and second, to show how the added standard (non-vanishing) diffusion affects the final solution.

The figure 3 shows the graphs of the time evolution of the $\left\|\Delta \tau^{n}\right\|$ obtained for various $\alpha(t)$. We can observe that for all cases the norm increases with the (pseudo) time towards an asymptotic value corresponding to the original non-diffusive model. It demonstrates that the added diffusion smoothes the solution and thus reduces the overall values of the solution Laplacian. Unless we completely remove (let vanish) the diffusion, the obtained solution doesn't corresponds to the original model.
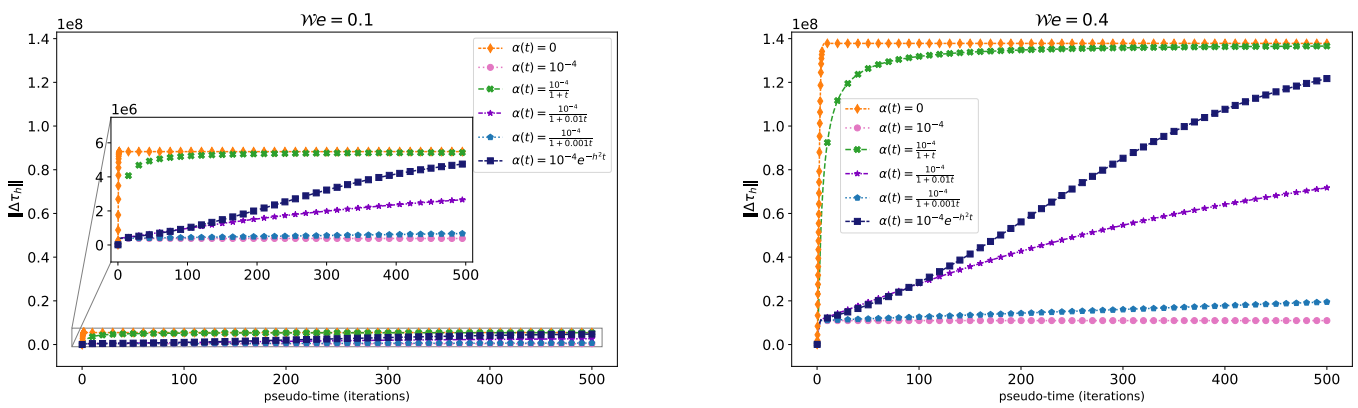

Figure 3: Comparison of $\left\|\Delta \boldsymbol{\tau}^{n}\right\|$ for severals $\alpha(t)$ for $\mathcal{W} e=0.1$ (left) and $\mathcal{W} e=0.4$ (right).

The figure 4 shows the graphs of the norm of the whole diffusive term $\left\|\alpha(t) \Delta \boldsymbol{\tau}^{n}\right\|$ for different $\alpha(t)$. We can observe that even though $\alpha(t)$ decays it doesn't means that the same happens for $\left\|\alpha(t) \Delta \boldsymbol{\tau}^{n}\right\|$, as the case of $\alpha(t)=\frac{10^{-4}}{1+0.001 t}$. This is also one of the reasons why the sufficient decay of $\alpha(t)$ is so important.
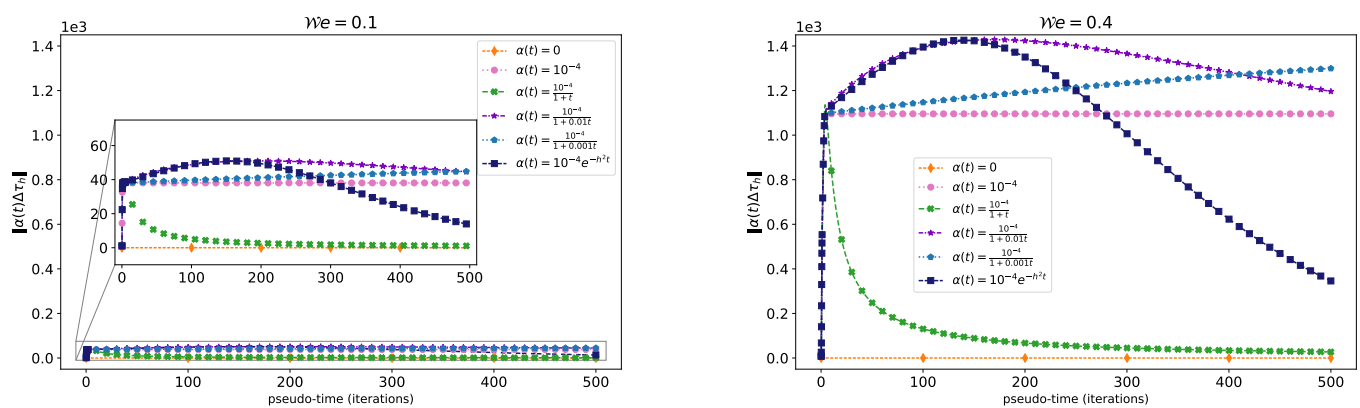

Figure 4: Comparison of $\left\|\alpha(t) \Delta \boldsymbol{\tau}^{n}\right\|$ for severals $\alpha(t)$ for $\mathcal{W} e=0.1$ (left) and $\mathcal{W} e=0.4$ (right). 
The main differences between the solutions with different levels of diffusion are apparent from the Fig. 5 showing the individual stress tensor components along the channel wall for two different Weissenberg numbers.

$\mathcal{W} e=0.1$
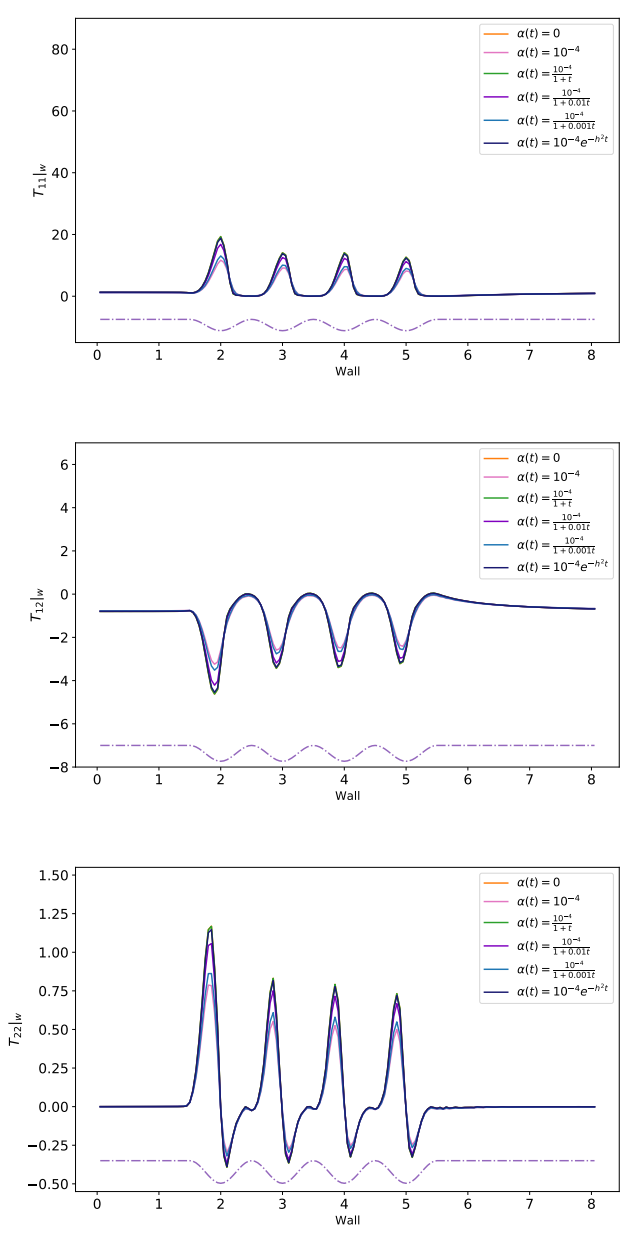

$\mathcal{W} e=0.4$
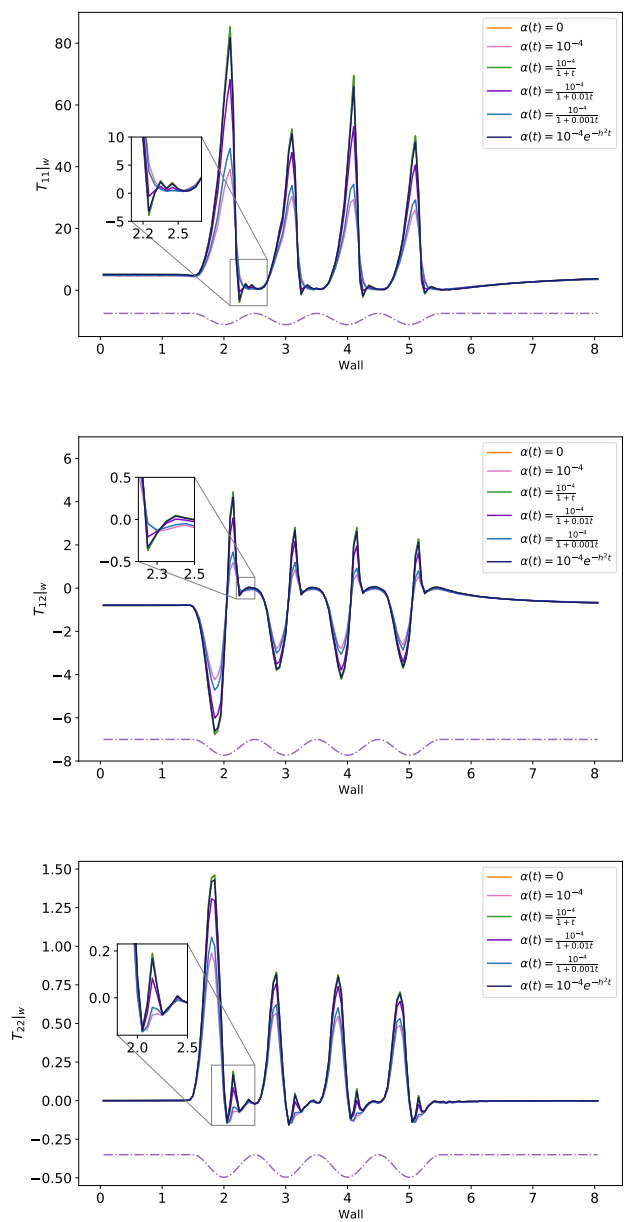

Figure 5: Components of $\boldsymbol{\tau}^{n}$ along the channel wall for different $\alpha(t)$.

From the Fig. 5 it is evident that when the diffusion is reduced, the stress peaks become larger and some more peaks can appear, compared to the highly diffusive solution obtained for the constant (non-vanishing) numerical diffusion. The minimal and maximal values of stress components are shown in the Fig. 6.
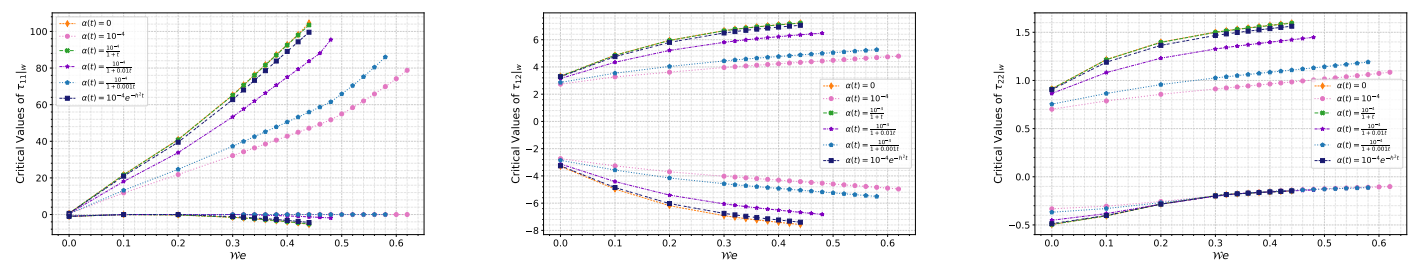

Figure 6: The maximum and minimum values of $\tau^{n}$ along the wall for various $\alpha(t)$.

Similar information can be deduced from the plots of the tension on the wall in Fig. 7. Again the wall stress tension is apparently larger with smaller diffusion coefficient $\alpha(t)$. The same tendency 

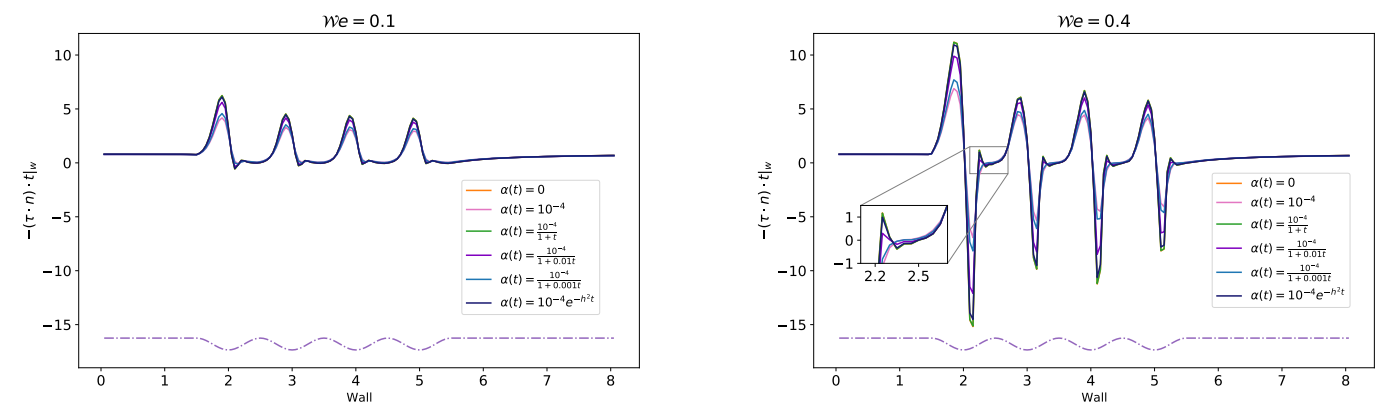

Figure 7: Comparison of stress tension along the wall for different $\alpha(t)$.

is confirmed in Fig. 8
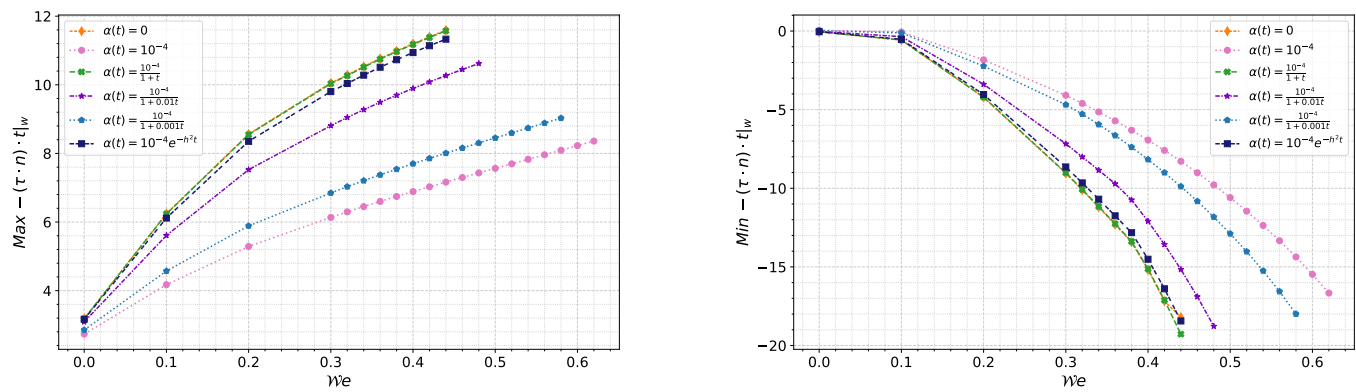

Figure 8: Comparison of stress tension for different $\alpha(t)$ depending on Weissenberg number.

\section{Conclusions and Remarks}

The initial series of numerical simulations presented in this paper shows the importance of the vanishing numerical diffusion. Although the added diffusion stabilizes the numerical model, it heavily affects the final solution. Thus the use of constant (non-vanishing) numerical diffusion is quite dangerous and should be used with extreme caution. The following conclusions and remarks can be drawn based on our simulations:

- The monotonically in time decaying numerical diffusion coefficient helps to stabilize the initial stage of the iterative process, without affecting the final solution. In this respect, this vanishing diffusion is much safer to use than the constant diffusion.

- The rate of decay for $\alpha(t)$ needs to be adjusted and optimized to allow the diffusion coefficient to completely vanish before the simulation is stopped (considered converged).

- Some simulations showed sudden temporal increase of residuals during the iterations. Such problems can't be stabilized by monotonically decreasing diffusion. In this respect the more complicated diffusion with time-derivative of Laplacian seems to work better.

Further work will focus on other optimized functions $\alpha(t)$. One of the possible improvements is to use the variable coefficient $\alpha$ depending on (pseudo) time-derivative of the quantity to be stabilized, i.e. $\alpha\left(\boldsymbol{\tau}_{t}\right)$. Such time-derivative dependent diffusion coefficient $\alpha$ will naturally vanish for steady state, while being able to self-adjust for sudden residual increase during the iteration process.

\section{Acknowledgment}

The financial support for the present project was partly provided by the Project FCT - Project FCT UIDB/04674/2020 and partly by the Czech Science Foundation under the grant No. P201-19-04243S. 


\section{References}

[1] Arada, N., Pires, M., \& Sequeira, A.: Numerical simulations of a shear-thinning Oldroyd-B fluids in curved pipes.IASME Transactions, Vol. 2, no. 6: (2005) pp. 948-959.

[2] Arada, N., Pires, M., \& Sequeira, A.: Numerical approximation of a viscoelastic Oldroyd-B flows in curved pipes. Kyoto Conference on the Navier-Stokes Equations and their Applications. RIMS Kôkyûroku Bessatsu, vol. B1: (2007) pp. 43-70.

[3] Arada, N., Pires, M., \& Sequeira, A.: Viscosity effects on flows of generalized Newtonian fluids through curved pipes. Computers and Mathematics with Applications. Vol. 53: (2007) pp. $625-646$.

[4] Bodnár, T., Pires, M. \& Janela, J.: Blood flow simulation using traceless variant of JohnsonSegalman viscoelastic model. Math. Model. Nat. Phenom. Vol. 9, no. 6: (2014) pp. 117-141.

[5] Girault, V. \& Raviart, P.-A.: Finite Element Methods for the Navier-Stokes Equations. Springer, Heidelberg(1986).

[6] Groisman, A. \& Steinberg, V.: Mechanism of elastic instability in Couette flow of polymer solutions. Experiment Phys. Fluids, vol. 10: (1998) pp. 2451-2463.

[7] Hecht, F.: New development in FreeFem++. Journal of numerical mathematics, vol. 20, no. 34: (2012) pp. 251-266.

[8] Hecht, F. \& Danaila, I.:FreeFem++ Lessons 5-8. https://www.ljll.math.upmc.fr/ hecht/ftp/ff++/2016-Fields/FH-Lesson5-8-Fields.pdf (2016).

[9] Marion, M. \& Temam, R.: Navier-Stokes Equations: Theory and Approximation. Handbook of Numerical Analysis vol. VI: (1998).

[10] Oldroyd, J.: Non-Newtonian effects in steady motion of some idealized elasto-viscous liquids. Proc. R. Soc. A. 245: (1958) pp. 278-297.

[11] Owens, R. \& Phillips, T. : Computational Rheology. Imperial College Press, London (2002).

[12] Pires, M. \& Sequeira, A.: Flows of Generalized Oldroyd-B Fluids in Curved Pipes. In: Escher J. et al. (eds) Parabolic Problems. Progress in Nonlinear Differential Equations and Their Applications, Springer, Basel, Vol. 80: (2011) pp. 21-43.

[13] M. Pires \& T. Bodnár, On the influence of diffusion stabilization in Oldroyd-B fuid flow simulations, in: Topical Problems of Fluid Mechanics 2020, 505 Institute of Thermomechanics CAS, Prague, 2020, pp. 176-183.

[14] Quarteroni, A. \& Valli, A.: Numerical Approximation of Partial Differential Equations Springer Publishing Company, Incorporated 2nd ed (2008).

[15] Rannacher, R.: Finite Element Methods for the Incompressible Navier-Stokes Equations. Lecture notes (1999). 